\title{
Isolation and structural determination of a new antibacterial compound demethyl-L-681,217 from Streptomyces cattleya
}

\author{
Shogo Sugai ${ }^{1}$, Hisayuki Komaki ${ }^{2}$, Hikaru Hemmi ${ }^{3}$ and Shinya Kodani ${ }^{1,4,5}$ \\ The Journal of Antibiotics (2016) 69, 839-842; doi:10.1038/ja.2016.53; published online 25 May 2016
}

Secondary metabolites including polyketides, nonribosomal peptides and hybrid polyketide-peptides have diverse chemical structures and a wide variety of bioactivities. ${ }^{1}$ Polyketides and nonribosomal peptides are synthesized by large enzyme complexes such as polyketide synthases (PKS) and nonribosomal peptide synthetases (NRPS). ${ }^{2}$ These biosynthetic proteins are typically encoded in neighboring loci and organized in gene clusters ranging from several to over $200 \mathrm{~kb}$ in length. NRPS and PKS employ a very similar strategy like an assembly line for the biosynthesis of two distinct classes of natural products. ${ }^{3-5}$ In addition, NRPS/PKS hybrid biosynthetic systems give structurally more diverse compounds by the combination of NRPS and PKS like building blocks on one assembly line. ${ }^{6}$

The biosynthetic gene cluster of a polyketide kirromycin ${ }^{7}$ (3, Figure 1b) was identified from the genome sequence of Streptomyces collinus ${ }^{8-10}$ and kirromycin was indicated to be biosynthesized by a large hybrid PKS/NRPS gene cluster. Among the related compounds including efrotomycin, ${ }^{11}$ dihydromocimycin, ${ }^{12}$ heneicomycin, ${ }^{13}$ factumycin ${ }^{14}$ and kirromycin, ${ }^{15}$ only the biosynthesis of kirromycin has been reported so far. ${ }^{8-10}$ Previously, a new kirromycin analog L-681,217 (2, Figure 1a), which lacked pyridine ring was isolated from Streptomyces cattleya ATCC 39203. ${ }^{16}$ On the basis of these results, we performed chemical investigation on a $\mathrm{MeOH}$ extract of S. cattelya NBRC 14057 (type strain) to search for new kirromycin analogs. As a result, a new kirromycin analog demethyl-L-681,217 (1) was isolated as an antibacterial compound from S. cattelya along with a known compound L-681,217 (2). As the genome sequence of the type strain of $S$. cattletya was previously determined, ${ }^{17}$ the biosynthetic gene cluster was searched for and found based on the similarity to that of kirromycin. Here we describe the isolation and structure determination of a new kirromycin analog demethyl-L-681,217 (1) and discuss the possible biosynthetic gene cluster.

The cultivation of $S$. cattleya NBRC 14057 was performed with 51 of ISP2 agar media. After 7 days of cultivation, cells of spore and aerial hyphae were collected and extracted with $\mathrm{MeOH}$. The $\mathrm{MeOH}$ extract was subjected to a CHP-20P column, washed with $60 \% \mathrm{MeOH}$, and eluted with $80 \% \mathrm{MeOH}$. The $80 \% \mathrm{MeOH}$ fraction was repeatedly subjected to preparatory HPLC to yield a new compound 1, along with a known compound 2 (Supplementary Figure S1).

The compound $\mathbf{1}$ was isolated as a white amorphous powder. High resolution ESI-TOF-MS analysis of 1 gave an $[\mathrm{M}+\mathrm{H}]^{+}$ion at $\mathrm{m} / \mathrm{z}$ 668.3372 (Supplementary Figure S2), consistent with a molecular formula of $\mathrm{C}_{35} \mathrm{H}_{51} \mathrm{NO}_{10}$ (calcd $[\mathrm{M}+\mathrm{H}]^{+}$ion at $\mathrm{m} / \mathrm{z}$ 668.3410). The molecular formula of 2 was also confirmed to be $\mathrm{C}_{36} \mathrm{H}_{53} \mathrm{NO}_{10}$ (Supplementary Figure S3) in the same manner. To obtain further information on the chemical structure, NMR experiments including ${ }^{1} \mathrm{H},{ }^{13} \mathrm{C}$, DEPT-135, HSQC, HMBC, DQF-COSY, NOESY and TOCSY spectra of 1 and 2 were performed in acetone- $d_{6}$ (Supplementary Figure S4). Identification of the structure of a known compound 2 was performed by 2D NMR spectra (Figure 1d). Briefly, five partial structures were indicated by five proton-proton spin systems (bold line, 2 in Figure 1d), established by TOCSY and DQF-COSY spectra. The HMBC correlations from H36 to C14, C15 and C16 indicated the connection between the partial structure of $\mathrm{C} 2-\mathrm{C} 14$ and that of $\mathrm{C} 16-\mathrm{C} 19$. The HMBC correlation from H16-C14 also supported this connection. The presence of a carboxyl residue at $\mathrm{C} 1$ was confirmed by $\mathrm{HMBC}$ correlation from $\mathrm{H} 2-\mathrm{C} 1$. The methoxy residue was assigned as attached at $\mathrm{C} 14$ by the $\mathrm{HMBC}$ correlation from $\mathrm{H} 35$ to $\mathrm{C} 14$. The HMBC correlations from H19 and H37 to C21 indicated the connection between the partial structure of C16-C19 and that of C22-C37-C38. The HMBC correlations from H22 and $\mathrm{H} 24$ to $\mathrm{C} 23$ indicated the connection between partial structure of C22-C37-C38 and that of C24-C25. The HMBC correlations from $\mathrm{H} 25$ and $\mathrm{H} 27$ to $\mathrm{C} 26$ indicated the connection between partial structure of C24-C25 and that of C27-C33. The NOESY correlation between $\mathrm{H} 25$ and $\mathrm{H} 27$ also supported this connection. The methyl residue was indicated as attached to $\mathrm{C} 26$ by the $\mathrm{HMBC}$ correlations from $\mathrm{H} 39$ to $\mathrm{C} 25, \mathrm{C} 26$ and C27. The presence of a tetrahydropyran

${ }^{1}$ Graduate School of Integrated Science and Technology, Shizuoka University, Shizuoka, Japan; ${ }^{2}$ Biological Resource Center, National Institute of Technology and Evaluation (NBRC), Kisarazu, Chiba, Japan; ${ }^{3}$ Food Research Institute, National Agriculture and Food Research Organization (NARO), Tsukuba, Ibaraki, Japan; ${ }^{4}$ College of Agriculture, Academic Institute, Shizuoka University, Shizuoka, Japan and ${ }^{5}$ Graduate School of Science and Technology, Shizuoka University, Shizuoka, Japan Correspondence: Dr S Kodani, College of Agriculture, Academic Institute, Shizuoka University, 836 Ohya, Suruga-ku, Shizuoka 422-8529, Japan.

E-mail; kodani.shinya@shizuoka.ac.jp

Received 7 February 2016; revised 12 April 2016; accepted 14 April 2016; published online 25 May 2016 


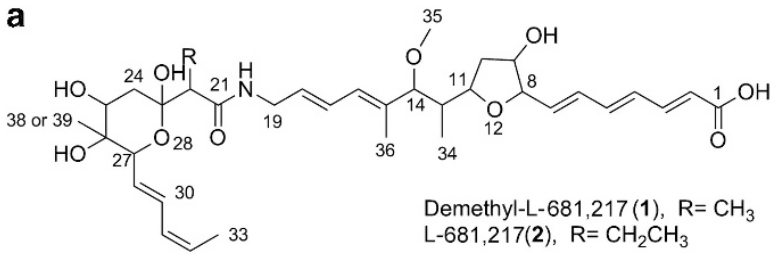

b

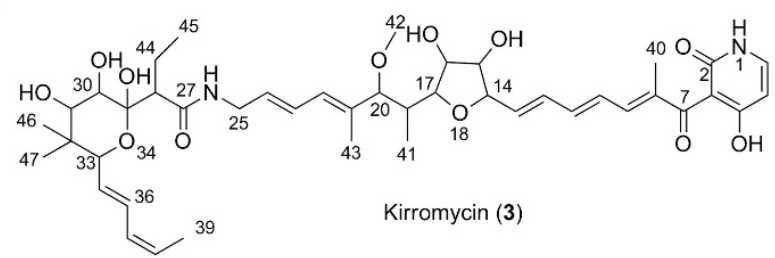

c

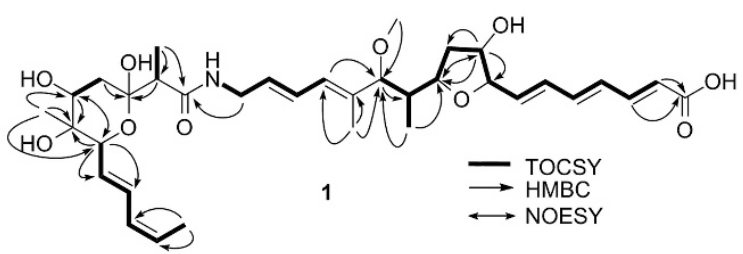

d

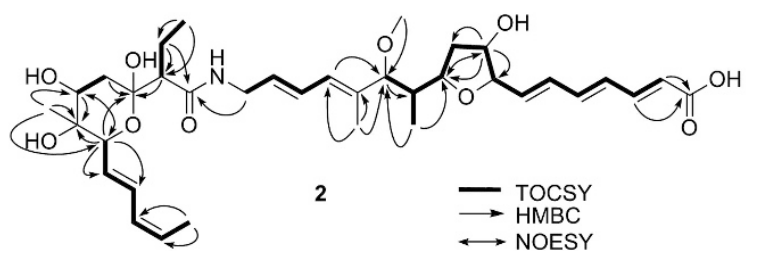

Figure 1 (a) Chemical structures of demethyl-L-681,217 (1) and L-681,217 (2); (b) Chemical structure of kirromycin (3); (c) Key 2D NMR correlations for structure determination of $\mathbf{1}$; (d) Key 2D NMR correlations for structure determination of $\mathbf{2}$.

ring was confirmed by HMBC correlation from $\mathrm{H} 27$ to C23. Regarding the assignment of tetrahydrofuran ring, a NOESY correlation between $\mathrm{H} 8$ and $\mathrm{H} 11$ was not observed, although a NOESY correlation between $\mathrm{H} 9$ and $\mathrm{H} 11$ was observed. In comparison with the reported NMR data of the related compound, ${ }^{18}$ the similarity of the chemical shifts indicated the presence of tetrahydrofuran ring.

The structure determination of $\mathbf{1}$ was accomplished by the comparison with the NMR data of 2 (Supplementary Table S1). Judging from the molecular weight (Supplementary Figures S2 and S3), the discrepancy between 1 and $\mathbf{2}$ was expected to be one methylene residue. As a result of interpretation of NMR data, the chemical shifts of $\mathbf{1}$ and $\mathbf{2}$ were almost identical except for a methyl and a methylene residue (Supplementary Table S1). Methyl residue ( $\delta \mathrm{H} 0.94$, triplet) and methylene residue ( $\delta \mathrm{H} 1.63$ and 1.72, multiplet) were lacking in the NMR spectrum of 1 and new methyl residue ( $\delta \mathrm{H} 1.20$, doublet) was observed in the NMR spectrum of 1 . The C37 methyl group $(\delta \mathrm{H} 1.20$, doublet) was connected to $\mathrm{C} 22$ by correlation of HMBC (Figure 1c). In the same manner with 2 , five proton-proton spin systems (bold line, $\mathbf{1}$ as shown in Figure 1c) were established by TOCSY and DQF-COSY spectra. The connections of the smaller spin systems were established by HMBC correlations shown in Figure 1c (one way arrows). Regarding the assignment of the tetrahydropyran ring, $\mathrm{HMBC}$ correlation from $\mathrm{H} 27$ to $\mathrm{C} 23$ was not observed.
The proton and carbon chemical shifts at position 8, 11, 23, 27 were almost identical with those of L-681,217 (Supplementary Table S1). Thus, we concluded the structure to be 1 as shown in Figure 1a.

The antimicrobial activities were measured by using a paper disk diffusion assay against bacterial strains (Escherichia coli, Pseudomonas aeruginosa, Serratia marcescens, Bacillus subtilis, Staphylococcus aureus, Micrococcus luteus, Streptomyces antibioticus), yeast strains (Saccharomyces cerevisiae, Schizosaccharomyces pombe, Kloeckera apiculata), and fungal strains (Aspergillus niger, Aspergillus oryzae, Mucor hiemalis). At the dosage of $10 \mu \mathrm{g}$ per disk, 1 and 2 showed the inhibitory zones of $13 \mathrm{~mm}$ and $18 \mathrm{~mm}$ in diameter, respectively, only against $S$. antibioticus. Interestingly, neither compound showed any antimicrobial activity against other tested microorganisms under these conditions.

As the complete genome sequence of S. cattleya NRRL 8057 (NBRC 14057) is available publicly, ${ }^{17}$ we searched for the loci encoding the biosynthesis of the two compounds L-681,217 and demethyl-L-681,217 (L-681,217s) in the genome sequence, and consequently found a large hybrid PKS/NRPS gene cluster shown in Supplementary Table S2. This cluster encoded five modular PKSs lacking internal AT domains (trans-AT type PKSs), two acyltransferases, one NRPS, two cytochrome P450s and one methyltransferase, which show high-sequence homologies to enzymes for kirromycin synthesis. ${ }^{10}$ Because L-681,217s are structurally related to kirromycin and the genome of $S$. cattleya NRRL 8057 harbors no other such gene cluster, we assumed this to be the biosynthetic gene cluster for L-681,217s and then bioinformatically analyzed. The module and domain organization of the hybrid PKS/NRPS gene cluster is shown in Figure 2. This cluser harbors 15 modules, which are very similar to 15 of 17 modules present in the kirromycin-synthetic gene cluster. ${ }^{10}$ According to the collinearity rule of the modular PKS/NRPS assembly line $^{6}$ and the similarity to kirromycin biosynthetic pathway, we predicted the chemical structure of the backbone synthesized by these enzymes. The domain organization of these PKS and NRPS proteins well agreed with the backbone structures of L-681,217s except for the difference at C25. As module 4 has a DH-KR pair as the optional domain, the $\mathrm{C}-\mathrm{C}$ bond between $\mathrm{C} 25$ and $\mathrm{C} 26$ would be double in L-681,217s, but it is actually single and C25 has a hydroxyl group. Hence, we assumed the DH domain in module 4 is inactive. The analysis of the PKS and NRPS module organization indicates that the biosynthesis starts with loading of an acetyl-CoA to SCAT_3576. The polyketide chain is extended by the PKS modules (modules 1-5) of SCAT_3576 and SCAT_3577, then condensed with glycine by the hybrid NRPS/PKS SCAT_3578, and further extended by SCAT_3579 to SCAT_3581. During or after release of the linear precursor of L-681,217s, this intermediate must undergo cyclization to yield two intramolecular rings and modifications. O-methylation of the keto group at $\mathrm{C} 14$ is presumably catalyzed by the methyltransferase of SCAT_3585. Cytochrome P450 of SCAT_3583 or SCAT_3588 is predicted to add an oxygen molecule at C26, yielding a hydroxyl group. Demethyl-L-681,217 is produced if module 5 loads methylmalonyl-CoA, whereby L-681,217 is produced if module 5 loads ethylmalonyl-CoA. However, the situation appears to be more complex than that, as two discrete acyltransferases are encoded as SCAT_3584 and SCAT_3586, respectively. SCAT_3584 contains two acyltransferase (AT) domains whose substrates are predicted to be malonyl-CoA. In contrast, SCAT_3586 contains only a single AT domain, which is predicted to recognize methylmalonyl-CoA as the substrate by the analysis using antiSMASH. ${ }^{19}$ Like KirCI and KirCII for kirromycin, one of the acyltransferases may be responsible for loading the ACPs needed for extension of the polyketide chain with 

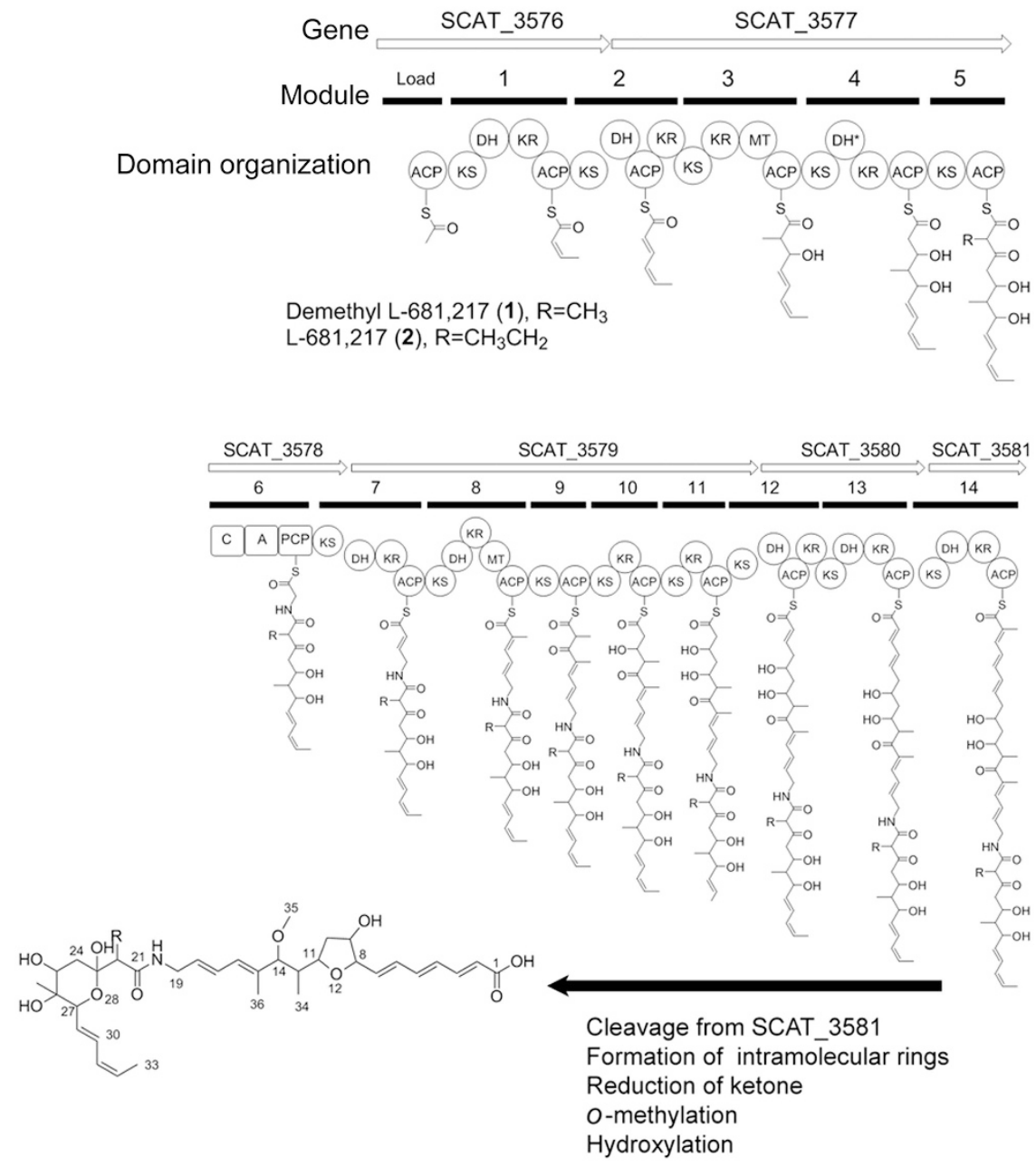

Figure 2 Proposed biosynthetic pathway of demethyl-L-681,217 (1) and L-681,217 (2). A, adenylation; ACP, acyl carrier protein; C, condensation; DH, dehydratase; $\mathrm{DH}^{*}$, inactive $\mathrm{DH}$; KS, ketosynthase; KR, ketoreductase; MT, methyltransferase; PCP, peptidyl carrier protein.

malonyl-CoA, whereas the other may catalyze the loading the ACPs of modules 5, 9 and 14 with alkylmalonyl-CoA such as methylmalonylCoA or ethylmalonyl-CoA. Although each of C15 and C24 also has a methyl side chain, these methyl groups are not derived from incorporation of methylmalonyl-CoA, but modules 3 and 8 incorporate malonyl-CoA molecules in the elongating polyketide chain and then the methyltransferase (MT) domains in these modules methylate the positions. Above all, we concluded that this gene cluster is responsible for the synthesis of L-681,217s and proposed the biosynthetic pathway, although genetic experiments such as gene disruption are needed to completely prove it.

\section{EXPERIMENTAL PROCEDURE}

\section{Bacterial strains}

The producing strain S. cattleya NBRC 14057 and test microorganisms (bacterial strains including, E. coli NBRC 102203, P. aeruginosa NBRC 12689, S. marcescens NBRC 102204, B. subtilis NBRC 13719, S. aureus NBRC 100910, M. luteus NBRC 3333, S. antibioticus NBRC 3117, yeast strains including S. cerevisiae NBRC 2376, S. pombe NBRC 0340, K. apiculata NBRC 0154; fungal strains including A. niger NBRC 33023, A. oryzae NBRC 4290, M. hiemalis NBRC 9405) for antimicrobial assays were obtained from the NBRC culture collection (NITE Biological Resource Center, Chiba, Japan).

\section{Isolation of demethyl-L-681,217}

S. cattleya NBRC 14057 was cultured using 51 of ISP2 agar medium for 7 days at $30^{\circ} \mathrm{C}$. The aerial hyphae and spore cells on the agar surface were harvested with a steel spatula. Double volume of $\mathrm{MeOH}$ was added to the harvested cells, followed by filtration with paper filter (Whatman No. 1, GE Healthcare Life Sciences, Little Chalfont, UK). The $\mathrm{MeOH}$ extract was concentrated to an aqueous residue using a rotary evaporator. The aqueous residue was subjected to open column chromatography using hydrophobic resin CHP-20P (Mitsubishi Chemical, Tokyo, Japan), eluted with $10 \% \mathrm{MeOH}, 60 \% \mathrm{MeOH}$, $80 \% \mathrm{MeOH}$, and $100 \% \mathrm{MeOH}$. The $80 \% \mathrm{MeOH}$ fraction was concentrated, and subjected to HPLC separation using an ODS column $(4.6 \times 250 \mathrm{~mm}$, Wakopak Handy-ODS, Wako Pure Chemical Industries, Ltd., Osaka, Japan), with elution (linear gradient of $25-55 \%$ MeCN containing $0.05 \%$ trifluoroacetic acid at the flow rate of $1 \mathrm{ml} \mathrm{min}^{-1}$ for $20 \mathrm{~min}$, monitoring $220 \mathrm{~nm}$ absorbance) to yield $1.3 \mathrm{mg}$ of demethyl-L-681,217 and $2.0 \mathrm{mg}$ of L-681,217 at the retention times of 17.2 and $18.8 \mathrm{~min}$, respectively (Supplementary Figure S1).

\section{NMR experiments}

Two NMR samples were prepared by dissolving 1 or 2 in $500 \mu$ of acetone- $d_{6}$, respectively. $1 \mathrm{D}^{1} \mathrm{H},{ }^{13} \mathrm{C}, \mathrm{DEPT}-135$, and all $2 \mathrm{D}$ NMR spectra were obtained on a Bruker Avance800 spectrometer with quadrature detection (Bruker BioSpin, Billerica, MA, USA). The $1 \mathrm{D}^{1} \mathrm{H},{ }^{13} \mathrm{C}$, DEPT- 135 spectra were recorded at $25{ }^{\circ} \mathrm{C}$ with 11 p.p.m. for proton and 240 p.p.m. for carbon. The following $2 \mathrm{D}$ 
${ }^{1} \mathrm{H}$-NMR spectra were recorded at $25^{\circ} \mathrm{C}$ with 10 p.p.m. spectra widths in $t 1$ and $t 2$ dimensions in the phase sensitive mode by States-TPPI method: $2 \mathrm{D}$ DQF-COSY, recorded with 512 and 1024 complex points in $t 1$ and $t 2$ dimensions; 2D homonuclear TOCSY with MLEV-17 mixing sequence, recorded with mixing time of $120 \mathrm{~ms}, 512$ and 1024 complex points in $t 1$ and $t 2$ dimensions; 2D NOESY, recorded with mixing time of $300 \mathrm{~ms}, 256$ and 1024 complex points in $t 1$ and $t 2$ dimensions. $2 \mathrm{D}{ }^{1} \mathrm{H}-{ }^{13} \mathrm{C}$ HSQC and HMBC spectra were acquired at $25^{\circ} \mathrm{C}$ in the echo-antiecho mode or in the absolute mode, respectively. The ${ }^{1} \mathrm{H}-{ }^{13} \mathrm{C}$ HSQC and $\mathrm{HMBC}$ spectra were recorded with 1024 and 512 complex points for 12 p.p.m. in the ${ }^{1} \mathrm{H}$ dimension and 160 p.p. $\mathrm{m}$. in the ${ }^{13} \mathrm{C}$ dimension or for 10 p.p.m. in the ${ }^{1} \mathrm{H}$ dimension and 210 p.p.m. in the ${ }^{13} \mathrm{C}$ dimension, respectively, at a natural isotope abundance. All NMR spectra were processed using XWINNMR (Bruker BioSpin). Before FT, the shifted sinebell window function was applied to $t 1$ and $t 2$ dimensions except for the HMBC spectrum. All ${ }^{1} \mathrm{H}$ and ${ }^{13} \mathrm{C}$ dimensions were referenced to acetone- $d_{6}$ at $25^{\circ} \mathrm{C}$.

\section{ESI-MS experiments}

ESI-TOF-MS spectra were recorded using a JEOL JMS-T100LP mass spectrometer. For high-resolution ESI-TOF-MS analysis, reserpine was used as an internal standard for caliblation.

\section{Antimicrobial assays}

By using a paper disk diffusion assay (6 $\mathrm{mm}$ in diameter, thick type) in the same manner of our previous report, ${ }^{20}$ the antimicrobial activity of 1 and 2 was mesured against all the test microorganism. Compunds 1 and 2 was dissolved in $\mathrm{MeOH}$ at the concentration of $1 \mathrm{mg} \mathrm{ml}^{-1}$. After all the test microroganisms were inoculated onto ISP2 agar medium, paper disks ( $10 \mu \mathrm{g}$ each per disk) were placed onto the surface of the agar medium, and paper disk with $\mathrm{MeOH}(10 \mu \mathrm{l})$ was used as a negative control. After incubation for 2 days at $30^{\circ} \mathrm{C}$, the diameter of the inhibitory zone was measured for evaluation of antimicrobiol activity.

\section{CONFLICT OF INTEREST}

The authors declare no conflict of interest.

\section{ACKNOWLEDGEMENTS}

This study was supported by the Japan Society for the Promotion of Science by Grants-in-aids (grant number 25350964).
1 Wang, H., Sivonen, K. \& Fewer, D. P. Genomic insights into the distribution, genetic diversity and evolution of polyketide synthases and nonribosomal peptide synthetases. Curr. Opin. Genet. Dev. 35, 79-85 (2015).

2 Sundaram, S. \& Hertweck, C. On-line enzymatic tailoring of polyketides and peptides in thiotemplate systems. Curr. Opin. Chem. Biol. 31, 82-94 (2016).

$3 \mathrm{Du}, \mathrm{L}$. \& Shen, B. Biosynthesis of hybrid peptide-polyketide natural products. Curr. Opin. Drug Discov. Dev. 4, 215-228 (2001).

4 Du, L., Sanchez, C. \& Shen, B. Hybrid peptide-polyketide natural products: biosynthesis and prospects toward engineering novel molecules. Metab. Eng. 3, 78-95 (2001).

5 Du, L. et al. Hybrid peptide-polyketide natural products: biosynthesis and prospects towards engineering novel molecules. Genet. Eng. (NY) 25, 227-267 (2003).

6 Fischbach, M. A. \& Walsh, C. T. Assembly-line enzymology for polyketide and nonribosomal peptide antibiotics: logic, machinery, and mechanisms. Chem. Rev. 106, 3468-3496 (2006).

7 Dahn, U. et al. Stoffwechselprodukte von mikroorganismen. 154. Mitteilung. Nikkomycin, ein neuer hemmstoff der chitinsynthese bei pilzen. Arch. Microbiol. 107, 143-160 (1976).

8 Laiple, K. J., Hartner, T., Fiedler, H. P., Wohlleben, W. \& Weber, T. The kirromycin gene cluster of Streptomyces collinus Tu 365 codes for an aspartate-alpha-decarboxylase, KirD, which is involved in the biosynthesis of the precursor beta-alanine. J. Antibiot. (Tokyo) 62, 465-468 (2009).

9 Musiol, E. M. et al. Supramolecular templating in kirromycin biosynthesis: the acyltransferase KirCII loads ethylmalonyl-CoA extender onto a specific ACP of the trans-AT PKS. Chem. Biol. 18, 438-444 (2011).

10 Weber, T. et al. Molecular analysis of the kirromycin biosynthetic gene cluster revealed beta-alanine as precursor of the pyridone moiety. Chem. Biol. 15, 175-188 (2008).

11 Wax, R., Maises, W., Weston, R. \& Birnbaum, J. Efrotomycin, a new antibiotic from Streptomyces lactamdurans. J. Antibiot. (Tokyo) 29, 670-673 (1976).

12 Maehr, H., Leach, M., Williams, T. H. \& Blount, J. F. The chemistry of aurodox and related antibiotics. Can. J. Chem. 58, 501-526 (1980).

13 Zimmerman, S. B., Chalmers, J. H. Jr., Dewey, R. S., Stapley, E. O. \& Hernandez, S. Heneicomycin, a new antibiotic (A21A): fermentation, isolation, and antibacterial spectrum. J. Antibiot. (Tokyo) 32, 665-666 (1979).

14 Gullo, V. P. et al. Factumycin, a new antibiotic (A40A): fermentation, isolation and antibacterial spectrum. J. Antibiot. (Tokyo) 35, 1705-1707 (1982).

15 Thein-Schranner, I., Zahner, H., Hoppe, H. U., Hummel, I. \& Zeeck, A. Metabolic products of microorganisms. 209 Kirrothricin, a new member of the kirromycin-group. J. Antibiot. (Tokyo) 35, 948-956 (1982).

$16 \mathrm{Kempf}$, A. J. et al. L-681,217, a new and novel member of the efrotomycin family of antibiotics. J. Antibiot. (Tokyo) 39, 1361-1367 (1986).

17 Barbe, V. et al. Complete genome sequence of Streptomyces cattleya NRRL 8057, a producer of antibiotics and fluorometabolites. J. Bacteriol. 193, 5055-5056 (2011).

18 McDonald, L. A., Lotvin, J. A., Bailey, A. E. \& Carter, G. T. Biosynthesis of ganefromycin: results from blocked mutants and bioconversion experiments. J. Nat. Prod 61, 217-226 (1998).

19 Blin, K. et al. antiSMASH 2.0-a versatile platform for genome mining of secondary metabolite producers. Nucleic Acids Res. 41, W204-W212 (2013).

20 Kodani, S., Murao, A., Hidaki, M., Sato, K. \& Ogawa, N. Isolation and structural determination of a new macrolide, makinolide, from the newly isolated Streptomyces sp. MK-30. J. Antibiot. (Tokyo) 65, 331-334 (2012).

Supplementary Information accompanies the paper on The Journal of Antibiotics website (http://www.nature.com/ja) 